'Fundação Oswaldo

Cruz (Fiocruz), Instituto Nacional de Saúde da Mulher, da Criança e do Adolescente Fernandes Figueira (IFF) - Rio de Janeiro, Brasil. leila.adesse@gmail.com

2 Fundação Oswaldo Cruz (Fiocruz), Instituto Nacional de Saúde da Mulher, da Criança e do Adolescente Fernandes Figueira (IFF) - Rio de Janeiro, Brasil.

katiass@iff.fiocruz.br

3 Fundação Oswaldo Cruz (Fiocruz), Instituto Nacional de Saúde da Mulher, da Criança e do Adolescente Fernandes Figueira (IFF) - Rio de Janeiro, Brasil.

cbonan@iff.fiocruz.br

4 Fundação Oswaldo Cruz (Fiocruz), Instituto Nacional de Saúde da Mulher, da Criança e do Adolescente Fernandes Figueira (IFF) - Rio de Janeiro, Brasil.

vaniamf@iff.fiocruz.br

\section{Complicações do abortamento e assistência em maternidade pública integrada ao Programa Nacional Rede Cegonha}

\author{
Abortion complications and assistance care in a public maternity \\ integrated to the Rede Cegonha National Program
}

Leila Adesse' ${ }^{\mathbf{1}}$ Kátia Silveira da Silva ${ }^{\mathbf{2}}$, Claudia Bonan², Vania Matos Fonseca $\mathbf{4}$

RESUMO Objetivou-se descrever o perfil sociodemográfico, obstétrico e assistencial de todas as internações de abortamento em maio-agosto/2012, em emergência, em estudo transversal. Resultado: 20,5\% eram adolescentes, 79,5\% tinham mais de 20 anos de idade e 48,7\% tinham a pele parda; $84,7 \%$ foram abortos não especificados e o tempo foi apropriado entre a classificação de risco e a internação em $36,6 \%$ dos casos. Há mudanças no perfil das complicações, com redução das infecções e aumento na porcentagem de adolescentes (20,5\%). Na atenção diferenciada do Programa Rede Cegonha, a avaliação de risco acolheu 90\% das mulheres em 30 minutos. O sub-registro do processo de abortamento persiste pela condição de ilegalidade e por dilemas ético-morais dos profissionais.

PALAVRAS-CHAVES Aborto; Complicações; Assistência hospitalar; Sub-registro.

ABSTRACT It was aimed to describe the socio-demographic, obstetric and care profile of all in-door abortion hospitalizations from May-August/2012, in emergency, in cross-sectional study. Result: $20.5 \%$ were adolescents, $79.5 \%$ were over 20 years old and $48.7 \%$ were brown colored; $84.7 \%$ were unspecified abortions and the time between risk assessment and admission was appropriate in $36.6 \%$ of the cases. There are changes in the profile of complications, with reduction of infections and percentage increase of adolescent abortions (20,5\%). In the differentiated approach of The Rede Cegonha National Program the risk assessment hosted 90\% of women in 30 minutes. The under-registration of the abortion process remains due to lawlessness condition and to ethical and moral dilemmas of the professionals.

KEYWORDS Abortion; Complications; Hospital care; Under registration. 


\section{Introdução}

O abortamento é um tema relevante para a saúde pública, em face de sua representatividade entre as causas de mortalidade e morbidade materna. Mulheres grávidas nos países em desenvolvimento continuam morrendo devido a quatro principais causas: hemorragias severas pós-parto, infecções, distúrbios hipertensivos e abortos.

No Brasil, em 2011, foram registrados 77 óbitos por aborto no Sistema de Informação de Mortalidade (SIM), o que corresponde a $4,8 \%$ do total de óbitos maternos e a uma razão de mortalidade materna de três óbitos maternos em decorrência de aborto por cem mil nascidos vivos. No mesmo ano, o abortamento figura entre a terceira causa de ocupação de leitos obstétricos, depois do parto e outras complicações obstétricas (VICTORA ET AL., 2011).

Em nosso País, pelo Código Penal de 1940, a prática do aborto induzido é considerada ilegal, abrindo exceção para as gestações resultantes de estupro e casos de risco de morte para a mulher. Em maio de 2012, o Supremo Tribunal Federal deu sentença favorável à interrupção voluntária da gravidez em casos de má formação fetal incompatível com a vida, como se dá na anencefalia. Nos demais casos, quando as mulheres decidem por interromper a gravidez, têm que recorrer a práticas ilegais e sob condições consideradas ilícitas.

Em tal contexto, as interrupções podem ser realizadas por pessoas sem as habilidades técnicas necessárias e/ou em ambiente que careça de mínimos padrões sanitários, sendo conceituadas pela Organização Mundial da Saúde (OMS) como abortos inseguros (OMS, 2013). As desigualdades sociais se manifestam nas opções entre os procedimentos para a interrupção da gravidez: de um lado, mulheres que podem arcar com o custo financeiro e realizam a intervenção de maneira rápida e segura; de outro, aquelas que recorrem a métodos mais inseguros e insalubres, expondo-as a complicações relacionadas à saúde reprodutiva que podem levar à morte.
No entanto, a tipificação do aborto como um delito não desestimula as mulheres de realizá-lo, o que cria uma situação favorável para procedimentos de risco com importantes consequências: põem em risco sua saúde e sua vida, já que essas práticas estão, muitas vezes, à margem do controle sanitário; dificultam um maior conhecimento da situação epidemiológica e o desenvolvimento de programas e ações de saúde que abarquem integralmente o fenômeno, inclusive, com medidas preventivas; geram e reproduzem um ambiente cultural de culpa e estigma, que penaliza, antes de tudo, a mulher. Todos esses aspectos estão imbricados e se manifestam na atenção prestada às mulheres que procuram serviços públicos com complicações de abortamento (MARIUTI ET AL., 2010).

A situação de ilegalidade contribui para um sub-registro dos abortamentos entre as causas de morbidade e mortalidade materna nos serviços de saúde, bem como para a definição de sua magnitude no Brasil, fazendo com que os estudos e as políticas públicas lancem mão apenas de estimativas, a partir das internações por abortamento registradas no Sistema de Informações Hospitalares (SIH) do Sistema Único de Saúde (SUS), o que, ainda assim, contabiliza um milhão de casos por ano (ADESSE; MONTEIRO; LEVIN, 2008).

A qualidade do atendimento prestado pode interferir na evolução das complicações do aborto inseguro. Nos países em desenvolvimento, tais cuidados são deficientes devido ao uso de métodos e técnicas obstétricas como a curetagem, tida como desatualizada quando comparada com os medicamentos e a aspiração manual intrauterina, bem como a falta de investimentos e estrutura dos serviços de saúde e de pessoal capacitado para prestar assistência de qualidade e de baixo custo (SINGH ET AL., 2010). Uma parcela destas complicações pode evoluir para o óbito, o que configura uma violação dos direitos reprodutivos das mulheres, uma vez que a maioria dessas mortes é considerada evitável se existisse acesso a uma atenção 
pós-abortamento precoce e de qualidade (OMS, 2013). Dada a complexidade com a qual o tema é tratado - pela sua ilegalidade, por ser tabu cultural e pelos dissensos religiosos -, encontram-se poucos estudos epidemiológicos de âmbito nacional (MENEZES; AQUINO, 2009). O panorama dessa condição obstétrica tem sido construído a partir de pesquisas locais e estaduais sobre as complicações do abortamento e as condições em que as mulheres são atendidas nos serviços públicos (VIEIRA ET AL. 2007; FUSCO; ANDREONI; SILVA, 2008).

Com esse cenário, o estudo aqui apresentado tem como objetivo conhecer os tipos e as complicações advindas do abortamento, além dos aspectos da assistência prestada, o perfil sociodemográfico e a história reprodutiva das mulheres internadas. $\mathrm{O}$ presente artigo é parte integrante de um projeto que se destina a explorar as experiências e as representações de profissionais de saúde ao atenderem mulheres em abortamento.

\section{Método}

Trata-se de um estudo transversal, exploratório, cuja população foi constituída por um censo das mulheres atendidas em processo de abortamento e com indicação de internação, no período de maio-agosto de 2012, totalizando 117 atendimentos. Para o cálculo do tamanho amostral definiu-se como parâmetro a frequência esperada de abortos, de $13,5 \%$, baseada em dados do Departamento de Informática do Sistema Único de Saúde (Datasus)/morbidade hospitalar no município do Rio de Janeiro (RJ), no ano anterior, onde foram registrados 2.400 partos na unidade hospitalar, nível de confiança de 0,05 (alpha) e erro amostral =0,06. As mulheres foram selecionadas consecutivamente no período definido acima.

O campo de estudo foi uma unidade de emergência que possui uma maternidade integrada, a qual dispõe de 39 leitos, sendo 6 reservados para os casos de abortamento, não sendo este serviço referência para casos de abortamento previstos em lei. Uma especificidade do mesmo é que ele está inserido na Rede Cegonha Carioca (RC), que tem como uma de suas diretrizes básicas a avaliação e classificação de risco e vulnerabilidades das gestantes, seja para parto, gravidez ou abortamento, logo na chegada à unidade. Neste momento, a gravidade do quadro clínico é avaliada e é gerado um protocolo de tempo de espera para o atendimento médico e a internação. A porta de entrada da demanda foi o setor de emergência ou diretamente a maternidade. Na maternidade, as mulheres receberam o primeiro atendimento dado pela equipe do Programa Cegonha Carioca, que é composta por enfermeiras e técnicas de enfermagem, sendo realizados o acolhimento em local específico, a aferição dos sinais vitais e a classificação de risco. Em seguida, as mulheres foram liberadas ou internadas. $\mathrm{O}$ critério de inclusão no estudo foi ter recebido diagnóstico de abortamento na admissão na emergência, considerando abortamento os casos de perda fetal com Idade Gestacional (IG) inferior a 22 semanas, definida pela ultrassonografia ou pela Data da Última Menstruação, e os casos de feto com peso inferior a 500 gramas (BRASIL, 2011). Foram excluídas as interrupções devido à gravidez ectópica ou mola hidatiforme. As pacientes foram primeiramente identificadas a partir de um livro de registros específico para os abortamentos. Os prontuários foram separados logo após a alta médica e a revisão técnica feita pelo chefe da maternidade, e entregues à pesquisadora para a seleção das informações, segundo formulário de pesquisa previamente elaborado.

As fontes de dados da pesquisa foram os documentos de uso institucional presentes nos prontuários médicos, que incluíam: boletim de emergência; ficha do Programa Cegonha Carioca; ficha de abortamento; ficha de cirurgia; e as evoluções médicas e de enfermagem. Essas fontes foram usadas de forma complementar na pesquisa, já que não 
havia uniformidade nos registros, além de dados faltosos em vários campos dos prontuários. As variáveis estudadas foram distribuídas em três grandes grupos. $\mathrm{O}$ primeiro deles, sobre as características sociodemográficas, incluiu: faixa etária (categorizada como 10 a 19 anos, 20 a 29 anos, 30 a 39 anos e 40 anos ou mais); cor/raça; local de residência; ocupação (segundo categorias profissionais anotadas no prontuário); e características reprodutivas (histórico de paridade e de abortamento). Já o segundo grupo de variáveis apresentou as características do abortamento. Para classificar o tipo de aborto, utilizou-se o critério de desencadeamento do processo abortivo, sendo categorizado como aborto não especificado, quando não havia explicitação, no prontuário, quanto ao desencadeamento deste; aborto espontâneo, quando constava tal diagnóstico; e aborto induzido, quando era informada, no prontuário, a realização de alguma manobra abortiva por parte das mulheres, prévia à internação. Os tipos de aborto foram apresentados segundo o intervalo da Classificação Internacional das Doenças (CID) O002-O007.

A variável Idade Gestacional (IG) foi medida em semanas ou em dias decorridos após a Data da Última Menstruação (DUM) da mulher grávida. A variável causa da internação incluiu sangramento, infecção, dor no baixo ventre e outras.

Para a variável avaliação de risco, foram utilizados os registros feitos pela equipe do Programa Cegonha Carioca, em 2013, que seguem as mesmas diretrizes do Ministério da Saúde para verificar condições físicas (sinais vitais) e mentais (níveis de consciência) das mulheres atendidas, de modo a hierarquizar prioridades para a internação na maternidade. Esta escala distribui-se segundo cores, indo do mais grave para o de menor risco, considerando, então, que a cor vermelha indica emergência, com necessidade de atendimento imediato; a cor laranja significa muita urgência, e demanda atendimento rápido, em até 10 minutos; o amarelo, urgência com atendimento em até 60 minutos; o verde indica casos de menor urgência, que podem ser atendidos em até 120 minutos; e os azuis, não urgentes, 240 minutos.

O terceiro e último grupo de variáveis incluiu os tempos (entre a chegada à unidade e a avaliação de risco; entre a avaliação de risco e a internação; e de internação) e os procedimentos terapêuticos da assistência (técnicas para esvaziamento uterino).

A coleta de dados foi realizada com o auxílio de um instrumento elaborado e aplicado pela autora principal deste artigo. A pesquisa foi aprovada pelo Comitê de Ética em Pesquisa do Instituto Nacional de Saúde da Mulher, da Criança e do Adolescente Fernandes Figueira - Fundação Oswaldo Cruz (IFF/Fiocruz), através do protocolo CAAE 0063.0.008.000-11. Para a construção do banco de dados e a análise estatística foi utilizado o programa EpiInfo, versão 3.5.3.

\section{Resultados}

No período da pesquisa foram realizadas 791 internações na maternidade, e cerca de $15 \%$ foram por motivo de abortamento ( $n=117)$. A maioria do grupo estudado era composta por mulheres adultas, com mais de 20 anos $(79,5 \%)$, e por uma quantidade expressiva de adolescentes (20,5\%). As mulheres de cor parda representavam $48,7 \%$ da amostra, e $29,9 \%$ eram de cor branca, porém, cerca de $10 \%$ dos prontuários não informavam sobre este quesito. Dos atendimentos prestados, $92,3 \%$ foram a moradoras do município do Rio de Janeiro. As ocupações predominantes eram de funções que demandavam baixo/médio nível de escolaridade e, consequentemente, eram de baixo/médio nível de proventos, sendo que, em $25,6 \%$ do total dos prontuários, não houve registro a este respeito (tabela 1). Quanto ao histórico reprodutivo, pouco mais da metade das mulheres (54,7\%) tiveram pelo menos um parto anterior ao evento obstétrico do aborto, e $25 \%$ delas tinha história anterior de abortamento (tabela 1). 
Tabela 1. Características sociodemográficas e reprodutivas das mulheres atendidas em abortamento inseguro, em hospital público, no município do Rio de Janeiro. Maio-agosto/2012

\begin{tabular}{|c|c|c|}
\hline Características Sociodemográficas $(n=117)$ & $\mathbf{n}$ & $\%$ \\
\hline \multicolumn{3}{|l|}{ FAIXA ETÁRIA } \\
\hline 10 a 19 anos & 24 & $20,5 \%$ \\
\hline 20 a 29 anos & 46 & $39,3 \%$ \\
\hline 30 a 39 anos & 39 & $33,3 \%$ \\
\hline 40 anos ou mais & 8 & $6,8 \%$ \\
\hline \multicolumn{3}{|l|}{ RAC̣A/COR } \\
\hline Branca & 35 & $29,9 \%$ \\
\hline Preta & 12 & $10,3 \%$ \\
\hline Parda & 57 & $48,7 \%$ \\
\hline Outros & 1 & $0,9 \%$ \\
\hline Ignorado & 12 & $10,3 \%$ \\
\hline \multicolumn{3}{|l|}{ MUNICÍPIO DE RESIDÊNCIA } \\
\hline Rio de Janeiro & 108 & $92,3 \%$ \\
\hline Outros & 4 & $3,6 \%$ \\
\hline Ignorado & 5 & $4,3 \%$ \\
\hline \multicolumn{3}{|l|}{ OCUPAC̣ÃO } \\
\hline Ignorada & 30 & $25,6 \%$ \\
\hline Do lar & 21 & $17,9 \%$ \\
\hline Doméstica/diarista & 13 & $11,1 \%$ \\
\hline Estudante & 8 & $6,8 \%$ \\
\hline Serviços/comércio & 31 & $26,5 \%$ \\
\hline Outras & 14 & $12 \%$ \\
\hline Características Reprodutivas & $\mathbf{n}$ & $\%$ \\
\hline \multicolumn{3}{|l|}{ HISTÓRICO DE PARIDADE } \\
\hline 0 & 33 & $28,2 \%$ \\
\hline 1 & 31 & $26,5 \%$ \\
\hline 2 & 26 & $22,2 \%$ \\
\hline 3 & 16 & $13,7 \%$ \\
\hline 4 ou mais & 7 & $6 \%$ \\
\hline Ignorado & 4 & $3,4 \%$ \\
\hline \multicolumn{3}{|l|}{ HISTÓRICO DE ABORTO } \\
\hline 0 & 81 & $69,2 \%$ \\
\hline 1 & 20 & $17,1 \%$ \\
\hline 2 & 5 & $4,3 \%$ \\
\hline 3 & 1 & $0,9 \%$ \\
\hline 4 & 3 & $2,6 \%$ \\
\hline Ignorado & 7 & $6 \%$ \\
\hline Total & 117 & $100 \%$ \\
\hline
\end{tabular}

Nota: Elaboração própria 
Mesmo com ausência de dados em 46,2\% dos prontuários sobre a presença do acompanhante no momento da chegada ao hospital (dados encontrados somente em 63 casos), verificou-se que $39,7 \%$ de mulheres estavam acompanhadas pelos maridos e $11,5 \%$ pelas mães (dados não mostrados na tabela 1 ).

A tabela 2 aponta um número elevado $(84,6 \%)$ de mulheres com diagnóstico de aborto não especificado e 1,7\% de aborto espontâneo, além de $13,7 \%$ de abortos induzidos. Nos casos dos abortos classificados como induzidos, todas as mulheres informaram ter utilizado, previamente ao atendimento em uma unidade de saúde, o medicamento misoprostol (Cytotec ${ }^{\circledR}$ ) em doses de dois comprimidos por via oral e intravaginal, para tentativas de autoaborto.

Tabela 2. Aspectos relacionados ao aborto e à internação dos prontuários de mulheres atendidas em abortamento, no município do Rio de Janeiro. Maio-agosto/2012 ( $n=117$ )

\begin{tabular}{|c|c|c|}
\hline Aspectos & $f$ & $\%$ \\
\hline \multicolumn{3}{|l|}{ TIPO DE ABORTO } \\
\hline Aborto não especificado & 99 & $84,6 \%$ \\
\hline Aborto induzido pela mulher & 16 & $13,7 \%$ \\
\hline Aborto espontâneo & 2 & $1,7 \%$ \\
\hline \multicolumn{3}{|l|}{ CAUSA DA INTERNAC̣ÃO } \\
\hline Hemorragia & 72 & $61,5 \%$ \\
\hline Infecção & 5 & $4,3 \%$ \\
\hline Outra & 37 & $31,6 \%$ \\
\hline Ignorada & 3 & $2,6 \%$ \\
\hline \multicolumn{3}{|l|}{ AVALIAC̣ÃO DE RISCO } \\
\hline Vermelha & 5 & $4,3 \%$ \\
\hline Laranja & 6 & $5,1 \%$ \\
\hline Amarela & 57 & $48,7 \%$ \\
\hline Verde & 34 & $29,1 \%$ \\
\hline Azul & 1 & $0,9 \%$ \\
\hline Ignorado & 14 & $12,0 \%$ \\
\hline \multicolumn{3}{|l|}{ MANOBRAS ABORTIVAS PRÉ-INTERNAC̣ÃO } \\
\hline Ignoradas & 100 & $85,5 \%$ \\
\hline Medicamento & 15 & $12,8 \%$ \\
\hline Chá+Medicamento & 1 & $0,9 \%$ \\
\hline TOTAL & 117 & $100 \%$ \\
\hline
\end{tabular}

Nota: Elaboração própria 
Em relação à causa de internação, destacou-se a hemorragia em $61,5 \%$ dos casos. Nesta mesma variável, $31,6 \%$ correspondiam a internações classificadas como outras causas. Incluíram-se aqui mulheres com queixas, como atraso menstrual acompanhado de dor ou sangramento leve e esparso, ou, até mesmo, com regressão destes sinais e sintomas, mas portando exame Ultrassonográfico (US) recente, realizado em serviço privado ou público, com ausência de sinais de vitalidade fetal - teste de Batimentos Cardíacos Fetais (BCF) com resultado negativo - ou ausência de saco gestacional. Em relação ao exame ginecológico, na internação, encontraram-se registros da presença de sangramento vaginal discreto em quase a metade dos casos (47,8\%), número destoante dos $61,5 \%$ encontrados no momento de acolhimento e avaliação do risco. Não houve referência à presença de corpo estranho nos casos examinados ou, ainda, qualquer registro de choque séptico, abdômen agudo e/ou perfuração uterina, embora tenha havido necessidade de transfusão de sangue em três casos (dados não incluídos em tabela).

No momento da classificação de risco pela Rede Cegonha, poucos casos foram identificados como de grande gravidade $(4,3 \%$ classificados com a cor vermelha), sendo mais frequentes $(77,8 \%)$ os de risco moderado a leve (amarelo e verde). Também nesta etapa de avaliação de risco houve registro em 16 prontuários sobre manobras abortivas realizadas pelas mulheres (tabela 2).
Merece menção o fato de que, através da IG dos 105 prontuários com esta informação, identificou-se que $80,1 \%$ estavam na faixa do primeiro trimestre, com até 12 semanas de gestação (dados não apresentados em tabela).

A tabela 3 apresenta a avaliação de risco da assistência segundo os tempos medidos a partir da entrada no setor de emergência, passando pelo Acolhimento com Classificação de Risco (ACR) à internação na maternidade, até a alta. A informação do tempo entre a chegada e a avaliação de risco estava disponível em $76 \%$ dos prontuários e, nestes, cerca de 90\% das mulheres foram acolhidas em até 30 minutos, demonstrando que a RC tem configurado uma atenção diferenciada para mulheres nessas condições. Por outro lado, a classificação não garantiu que o seguimento do cuidado (internação ou não) acontecesse no tempo preconizado pelo programa da RC: a quase totalidade dos casos classificados como vermelho (imediato) e laranja (até 10 minutos) não teve encaminhamento para atendimento médico no tempo adequado. A média do tempo de internação para todas as mulheres, independentemente da classificação de risco inicial, foi de três dias e meio. Quando este dado é analisado pormenorizadamente, identifica-se que as de menor gravidade (cor verde) acompanham esta média, enquanto as de maior gravidade (cor vermelha) têm predominância de um período de internação total de dois dias.

Tabela 3. Avaliação de risco segundo os tempos da assistência de mulheres atendidas em abortamento em unidade pública no município do Rio de Janeiro. Maio-agosto/2012 ( $n=117)$

\begin{tabular}{|c|c|c|c|c|c|c|c|c|c|c|c|c|c|c|}
\hline \multirow[b]{3}{*}{$\begin{array}{l}\text { Tempo entre chegada } \\
\text { e avaliação de risco }\end{array}$} & \multicolumn{14}{|c|}{ AVALIAC̣ÃO DE RISCO } \\
\hline & \multicolumn{2}{|r|}{ Azul } & \multicolumn{2}{|r|}{ Verde } & \multicolumn{2}{|c|}{ Amarela } & \multicolumn{2}{|c|}{ Laranja } & \multicolumn{2}{|c|}{ Vermelha } & \multicolumn{2}{|c|}{ Ignorado } & \multicolumn{2}{|c|}{ Total } \\
\hline & $n$ & $\%$ & $\mathrm{n}$ & $\%$ & $\mathrm{n}$ & $\%$ & $\mathrm{n}$ & $\%$ & $n$ & $\%$ & $\mathrm{n}$ & $\%$ & $n$ & $\%$ \\
\hline Imediato & 0 & $0,0 \%$ & 6 & $17,6 \%$ & 9 & $15,8 \%$ & 2 & $33,3 \%$ & 3 & $60,0 \%$ & 0 & $0,0 \%$ & 20 & $17,1 \%$ \\
\hline 1 a 10 minutos & 1 & $100,0 \%$ & 14 & $41,2 \%$ & 27 & $47,4 \%$ & 4 & $66,7 \%$ & 1 & $20,0 \%$ & 1 & $7,1 \%$ & 48 & $41,0 \%$ \\
\hline 11 a 30 minutos & 0 & $0,0 \%$ & 8 & $23,5 \%$ & 4 & $7,0 \%$ & 0 & $0,0 \%$ & 0 & $0,0 \%$ & 0 & $0,0 \%$ & 12 & $10,3 \%$ \\
\hline 31 a 120 minutos & 0 & $0,0 \%$ & 2 & $5,9 \%$ & 7 & $12,3 \%$ & 0 & $0,0 \%$ & 0 & $0,0 \%$ & 0 & $0,0 \%$ & 9 & $7,7 \%$ \\
\hline Ignorado & 0 & $0,0 \%$ & 4 & $11,8 \%$ & 10 & $17,5 \%$ & 0 & $0,0 \%$ & 1 & $20,0 \%$ & 13 & $92,9 \%$ & 28 & $23,9 \%$ \\
\hline Total & 1 & $100,0 \%$ & 34 & $100,0 \%$ & 57 & $100,0 \%$ & 6 & $100,0 \%$ & 5 & $100,0 \%$ & 14 & $100,0 \%$ & 117 & $100,0 \%$ \\
\hline
\end{tabular}


Tabela 1. (cont.)

\begin{tabular}{|c|c|c|c|c|c|c|c|c|c|c|c|c|c|c|}
\hline $\begin{array}{l}\text { Tempo entre avaliação } \\
\text { de risco e internação }\end{array}$ & $n$ & $\%$ & $\mathrm{n}$ & $\%$ & $n$ & $\%$ & $n$ & $\%$ & $n$ & $\%$ & $n$ & $\%$ & $n$ & $\%$ \\
\hline Imediato & 0 & $0,0 \%$ & 1 & $2,9 \%$ & 1 & $1,8 \%$ & 0 & $0,0 \%$ & 0 & $0,0 \%$ & 1 & $7,1 \%$ & 3 & $2,6 \%$ \\
\hline 1 a 10 minutos & 0 & $0,0 \%$ & 0 & $0,0 \%$ & 4 & $7,0 \%$ & 1 & $16,7 \%$ & 1 & $20,0 \%$ & 0 & $0,0 \%$ & 6 & $5,1 \%$ \\
\hline 11 a 30 minutos & 1 & $100,0 \%$ & 4 & $11,8 \%$ & 6 & $10,5 \%$ & 2 & $33,3 \%$ & 2 & $40,0 \%$ & 0 & $0,0 \%$ & 15 & $12,8 \%$ \\
\hline 31 a 120 minutos & 0 & $0,0 \%$ & 12 & $35,4 \%$ & 19 & $33,3 \%$ & 2 & $33,3 \%$ & 1 & $20,0 \%$ & 0 & $0,0 \%$ & 34 & $29,1 \%$ \\
\hline 121 a 240 minutos & 0 & $0,0 \%$ & 5 & $14,7 \%$ & 9 & $15,8 \%$ & 0 & $0,0 \%$ & 0 & $0,0 \%$ & 1 & $7,1 \%$ & 15 & $12,8 \%$ \\
\hline $\begin{array}{l}\text { tempo excede } \\
\text { a regra }\end{array}$ & 0 & $0,0 \%$ & 6 & $17,6 \%$ & 3 & $5,3 \%$ & 0 & $0,0 \%$ & 0 & $0,0 \%$ & 0 & $0,0 \%$ & 9 & $7,7 \%$ \\
\hline Ignorado & 0 & $0,0 \%$ & 6 & $17,6 \%$ & 15 & $26,3 \%$ & 1 & $16,7 \%$ & 1 & $20,0 \%$ & 12 & $85,8 \%$ & 35 & $29,9 \%$ \\
\hline Total & 1 & $100,0 \%$ & 34 & $100,0 \%$ & 57 & $100,0 \%$ & 6 & $100,0 \%$ & 5 & $100,0 \%$ & 14 & $100,0 \%$ & 117 & $100,0 \%$ \\
\hline Tempo de internação & $n$ & $\%$ & $\mathrm{n}$ & $\%$ & $n$ & $\%$ & $n$ & $\%$ & $n$ & $\%$ & $\mathrm{n}$ & $\%$ & $n$ & $\%$ \\
\hline 1 & 0 & $0,0 \%$ & 4 & $11,8 \%$ & 9 & $15,8 \%$ & 1 & $16,7 \%$ & 0 & $0,0 \%$ & 2 & $14,3 \%$ & 16 & $13,7 \%$ \\
\hline 2 & 1 & $100,0 \%$ & 11 & $32,4 \%$ & 23 & $40,3 \%$ & 2 & $33,2 \%$ & 4 & $80,0 \%$ & 6 & $42,9 \%$ & 47 & $40,2 \%$ \\
\hline 3 & 0 & $0,0 \%$ & 9 & $26,5 \%$ & 13 & $22,8 \%$ & 1 & $16,7 \%$ & 0 & $0,0 \%$ & 3 & $21,4 \%$ & 26 & $22,2 \%$ \\
\hline 4 & 0 & $0,0 \%$ & 6 & $17,6 \%$ & 7 & $12,3 \%$ & 1 & $16,7 \%$ & 0 & $0,0 \%$ & 2 & $14,3 \%$ & 16 & $13,7 \%$ \\
\hline 5 ou mais & 0 & $0,0 \%$ & 3 & $8,8 \%$ & 4 & $7,0 \%$ & 1 & $16,7 \%$ & 1 & $20,0 \%$ & 1 & $7,1 \%$ & 10 & $8,5 \%$ \\
\hline Em branco & 0 & $0,0 \%$ & 1 & $2,9 \%$ & 1 & $1,8 \%$ & 0 & $0,0 \%$ & 0 & $0,0 \%$ & 0 & $0,0 \%$ & 2 & $1,7 \%$ \\
\hline Total & 1 & $100,0 \%$ & 34 & $100,0 \%$ & 57 & $100,0 \%$ & 6 & $100,0 \%$ & 5 & $100,0 \%$ & 14 & $100,0 \%$ & 117 & $100,0 \%$ \\
\hline
\end{tabular}

Nota: Elaboração própria

Outro dado observado nas fichas do centro obstétrico foi que o procedimento de esvaziamento uterino tem registro diferenciado: no caso de aborto retido (colo fechado e BCF negativo), era administrado o medicamento misoprostol para a dilatação do colo, seguido de curetagem (29,1\%); em outros atendimentos, havia a utilização de ocitocina venosa seguida de curetagem (66,7\%). Não houve uso da técnica de Aspiração Manual Intrauterina (Amiu), bem como não foram realizados esvaziamentos uterinos em cinco casos: duas mulheres saíram à revelia; duas o fizeram pela demora na realização do ultrassom, após três dias de internação; e uma, por expulsão total do feto (aborto completo).

\section{Discussão}

Este estudo observou uma proporção maior de adolescentes do que a que fora observada na década passada, e isto coincide com valores $(20,7 \%)$ de outra pesquisa, realizada na cidade de Bauru, em São Paulo (VIeIRA Et AL., 2007). Pesquisa nacional com dados do período de 1992-2005 aponta o grupo etário das adolescentes e as residentes na região Norte (ADESSE; MONTEIRO; LEVIN, 2008) como as únicas faixas em que a taxa anual de abortamento é de tendência crescente. Estes resultados corroboram a ideia de uma mudança no perfil etário dos casos de abortamento, em relação à década passada.

O relatório do Fundo de População das Nações Unidas (WEBER, 2012) destacou a ocorrência de 71 partos para cada mil adolescentes entre 15 e 19 anos, situando nosso País em $128^{\circ}$ no ranking de gravidez precoce, entre 188 países, e sugerindo a necessidade de conhecimento sobre o grau de vulnerabilidade das adolescentes, suas experiências sexuais, de contracepção e práticas de aborto. Tais dados reforçam um necessário deslocamento das políticas públicas das mulheres para um enfoque não apenas nas que já foram mães, como também nas que se iniciam na vida sexual (HEILBORN ET AL., 2011). Estudo internacional das taxas de aborto apresentadas 
por distribuição etária em diferentes países seguiram três padrões básicos (BANKOLE; SINGH; HAAS, 1999): um com a forma de U, em Cuba, no Canadá e na Dinamarca, com um declínio das taxas após a adolescência, entre mulheres de 20-24 anos ou 25-29 anos, e níveis de crescimento máximo entre 40 anos e mais; e outro, de aumento linear com a idade, na Bulgária, na Turquia e no Cazaquistão. Em países como a República Tcheca, na década de 1990, as taxas de aborto tiveram uma associação positiva com o aumento da idade. Já na Suécia e na Holanda, há um terceiro padrão: a curva é um $\mathrm{J}$ invertido quando as adolescentes têm uma taxa mais alta do que as mulheres da faixa etária de 40 anos e mais. Acrescentamos, analisando os dados do Datasus (ADESSE; MONTEIRO; LEVIN, 2008), que a curva no Brasil seria em forma de U com um crescimento na adolescência seguido de um declínio após 29 anos e um novo aumento depois dos 40 anos.

Pesquisa nacional (DINIZ;MEDEIROS, 2010) apontou que o abortamento é mais frequente entre mulheres com menor nível de escolaridade. Os efeitos diretos da baixa escolaridade podem estar expressos na falta de informação sobre sexualidade e reprodução, mas também afetam as condições de participação no mercado de trabalho, o acesso à renda e os padrões de união conjugal, entre outros. Por isso, a variável ocupação (26,5\% das mulheres com ocupação em serviços de comércio e 11,9\% de domésticas) foi utilizada como proxy da escolaridade. Poderíamos acrescentar que tais situações de vulnerabilidades imbricadas influenciam o acesso à assistência de saúde reprodutiva. Embora haja falta de registro nos prontuários sobre o nível de escolaridade, as ocupações desempenhadas pelas mulheres analisadas denotam que a maioria faz parte de grupos populacionais socialmente vulneráveis e estão inseridas em um contexto de desigualdades sociais.

Os registros dos prontuários pouco esclareciam sobre as circunstâncias do início do processo de abortamento: somente 2 casos foram anotados como abortamentos espontâneos, enquanto 16 (13,7\%) foram referidos pelas pacientes como induzidos por manobras abortivas pré-internação. Cabe lembrar que, das internações no Sistema Único de Saúde por motivo de abortamento, estima-se que, $25 \%$ destes sejam espontâneos; $2 \%$, abortos previstos em lei; e os demais, induzidOS (ADESSE; MONTEIRO; LEVIN, 2008).

A precocidade da ocorrência dos abortamentos refletida na IG abaixo de 12 semanas, para $93 \%(n=103)$ das mulheres, é um dado que pode ser considerado revelador, na medida em que os casos de IG avançada estão mais associados a complicações, conforme descrito em outras pesquisas (RAMOS; FERREIRA; SOUZA, 2010). Além disso, houve ausência de óbitos ou necessidade de intervenções cirúrgicas maiores ou de terapia intensiva, embora a média do tempo de internação das mulheres tenha sido de três dias.

A totalidade dos prontuários apresentou ausência de registro do diagnóstico de entrada e de saída, uma condição que pode estar presente em outros serviços do SUS que recebem mulheres em situação de abortamento e pode contribuir para o sub-registro do número e dos tipos de abortamentos existentes no País. Um importante achado da presente pesquisa diz respeito ao número elevado de mulheres com diagnóstico de aborto não especificado (84,6\%). A falta desse registro pode representar uma prática justificada, talvez, pela condição de ilegalidade dos abortos que chegam em curso nos serviços de saúde, através dos quais os profissionais vivenciam dilemas éticos, morais e/ou de objeção de consciência. Dessa forma, lança-se mão de estimativas e é legítima a suposição de que, entre os casos estudados, há uma grande proporção de abortamentos insegurOS (ADESSE; MONTEIRO; LEVIN, 2008).

Quanto às complicações do abortamento, o registro de infecção em apenas $4,3 \%$ dos casos, sem indicativo de choque, abdômen agudo e/ou perfuração uterina, aponta uma morbidade com bom prognóstico para as mulheres, ainda que possa ter havido sub-registros de possíveis manobras abortivas 
feitas pelas mulheres, e de complicações decorrentes destes abortamentos. Mesmo para os casos considerados mais graves, na avaliação de risco da porta de entrada (80\% das mulheres com risco de cor vermelha e 33,3\% de cor laranja), o tempo de permanência foi de dois dias de internação na unidade.

Apesar dos limites legais, algumas mulheres informaram - e os profissionais registraram - $\mathrm{o}$ uso de Cytotec ${ }^{\circledast}$ (misoprostol) para os abortos induzidos. Este dado, ainda que reduzido em nossa pesquisa, pode expressar uma mudança detendência das manobras abortivas realizadas pelas mulheres, com a substituição das antigas práticas populares (chás, aplicação in loco de permanganato de potássio e outras substâncias abrasivas, objetos perfurantes, intervenções de 'curiosas') pelo Cytotec ${ }^{\circledR}$ (SINGH; MONTEIRO; LEVIN, 2012). Pesquisas com enfoque nas complicações pós-abortamento, sobre o uso deste medicamento por mulheres, para indução, referem uma menor ocorrência de eventos infecciosos e hemorrágicos, diferentemente dos métodos descritos na década passada. A singularidade da experiência das mulheres brasileiras com o misoprostol tem sido expressa em publicações acadêmicas e leigas (MENEZES; AQUINO, 2009).

Em relação ao tratamento, enquanto o Ministério da Saúde preconiza a utilização da técnica de Amiu nos casos de esvaziamento uterino, em até 12 semanas de gestação (BRASIL, 2011), o procedimento de rotina nesta maternidade é a curetagem uterina. Segundo o mesmo guia técnico, são escolhas aceitáveis de método no primeiro trimestre: aspiração intrauterina, abortamento farmacológico (misoprostol) e curetagem uterina. Estudo de Saciloto et al. (2011) apontou que o procedimento Amiu pode reduzir o período de internação, sendo, inclusive, uma medida de verificação do fluxo e da qualidade da atenção. Também recomendado e progressivamente utilizado nos casos de abortamento, o misoprostol - análogo sintético da prostaglandina - tem ação uterotônica e de amolecimento do colo uterino. Este medicamento consta da $7^{a}$. Portaria da Relação Nacional de Medicamentos Essenciais (Rename) desde 2010, e teve protocolo de uso editado pelo Ministério da Saúde em 2012 (BRASIL, 2012).

Um aspecto interessante, que pode estar associado à implementação da RC, é a presença de acompanhantes $(53,84 \%)$ na chegada das pacientes à unidade. Componente da assistência ao parto e ao nascimento, esta proposta de modelo de atenção humanizada rompe com uma prática passada, de deixar a mulher sozinha como uma punição por ter interrompido a gravidez, e pode representar certo nível de apoio social (AQUINO ET AL., 2012). Destaca-se, também, a importância deste registro para que seja avaliado o cumprimento dessa diretriz no âmbito do SUS (Lei ${ }^{\circ}$ 11.108) e para que seja permitida a medição de seu efeito na humanização da assistência ao abortamento e na saúde da mulher.

Outra questão relacionada à RC foi a diretriz de acolhimento e avaliação de risco, que permitiu avaliar que, para a maioria dos casos mais graves, o tempo para a internação ultrapassou o limite indicado (RIO DE JANEIRO, 2013). Os dados relativos à classificação de cor vermelha indicaram que todos excederam o tempo preconizado para a internação imediata. Em relação aos de cor laranja, apenas um caso seguiu encaminhamento no tempo adequado.

\section{Conclusão}

Segundo estimativas da Organização Mundial de Saúde, metade das gestações são indesejadas, com uma a cada nove mulheres recorrendo ao abortamento (OMS, 2013). A preocupação com a redução da morbimortalidade por abortamento tem sido objeto de normas e protocolos do Ministério da Saúde à luz dos objetivos das Metas do Desenvolvimento do Milênio (BRASIL, 2010). Com base na observação empírica de que existe uma defasagem entre o preconizado e o realizado no SUS, deu-se a motivação deste estudo, bem como a necessidade de se 
conhecer os graus e tipos das complicações do abortamento e da assistência prestada nos serviços emergenciais de saúde.

As condições restritivas e punitivas da lei brasileira podem ser importantes justificativas para a ocorrência de limitações no acesso aos serviços de saúde com vistas ao atendimento precoce, principalmente nos casos com complicações obstétricas geradas pelo abortamento induzido. No entanto, a maioria dos registros quanto à IG inferior a 12 semanas pode ter contribuído para um perfil de complicações mais leves em nossos achados, corroborando a literatura científica (RAMOS; FERREIRA; SOUZA, 2010), na qual $o$ abortamento realizado em IG menos avançada estaria associado a menores índices de complicações. Daquelas mulheres que informaram como o aborto foi realizado, todas citaram o uso de misoprostol. As evidências científicas (BRASIL, 2012; OSAVA ET AL., 1997) têm ampliado o debate sobre a possibilidade de o acesso a este medicamento, pelas mulheres (ainda que usado em situação de ilicitude), possa reduzir a morbidade e a mortalidade por abortamento.

Pela leitura dos prontuários, pôde-se observar que, naquela unidade, $o$ atendimento às mulheres em abortamento é dissonante em relação ao preconizado pela Norma Técnica do Ministério da Saúde (BRASIL, 2010) quanto ao esvaziamento uterino com o procedimento de Amiu, este que implicaria em redução do tempo de permanência na unidade, uma vez que esta técnica demanda menor nível de anestesia e recuperação mais rápida (SACILOTO ET AL., 2011).

Cabe ressaltar a importância das iniciativas de aprimoramento da qualidade da assistência à gravidez, ao parto e ao puerpério por meio do Programa Cegonha Carioca (2013), semelhante ao programa nacional da RC. A inclusão dos casos de abortamento na proposta de acolhimento e avaliação de risco expressa uma mudança na direção de um modelo de atenção humanizada e baseado em evidências científicas, o que pode contribuir para a redução da mortalidade materna por esta causa. Os resultados do estudo indicam, porém, que ainda são necessários investimentos para que as ações previstas sejam realizadas de acordo com os protocolos assistenciais disponíveis. Isto porque somente $36,6 \%$ das mulheres que passaram pela avaliação de risco foram adequadamente encaminhadas, segundo o tempo preconizado.

$\mathrm{O}$ acolhimento orientado pelos princípios da humanização da assistência e de garantia de direitos humanos contempla "o tratamento digno e respeitoso, a escuta, o reconhecimento e a aceitação das diferenças, o respeito ao direito de decidir de homens e mulheres" (BRASIL, 2011, P. 23). É nesta etapa inicial do fluxo da atenção que, potencialmente, as barreiras operacionais de acesso à internação podem ser reduzidas, constituindo, assim, um espaço para uma escuta privilegiada, por exemplo, sobre os métodos utilizados em manobras abortivas por mulheres, no caso do abortamento induzido.

A proposta da RC possibilita privacidade e confidencialidade, e pode operar mudanças no processo do cuidado e do respeito aos direitos das mulheres. Com trato respeitoso, livre de julgamento moral e embasado nos critérios técnicos das vulnerabilidades clínico-obstétricas, as equipes de saúde podem contribuir para um tratamento mais adequado às necessidades da clientela, atendendo aos princípios da Política Nacional de Humanização da Atenção com acolhimento e classificação de risco em tempo adequado pelos serviços.

Os registros sobre aborto permanecem ocultos, impossibilitando o desvelamento da magnitude do tema pelas pesquisas de prontuários médicos. Isto talvez se justifique pela condição de ilegalidade dos abortos incompletos que chegam aos serviços de saúde, através dos quais os profissionais vivenciam dilemas éticos, morais e/ou de objeção de consciência. Em nosso estudo, a quase totalidade dos prontuários apresentava falta de anotação do diagnóstico de entrada e da CID, 
o que pode se refletir no sub-registro do número e dos tipos de abortamentos. A falta de informações em diversos campos dos prontuários foi um fator limitador no estudo, o que culminou na classificação da grande maioria dos tipos de abortamento (84,7\%) como 'não especificado'.

Ainda que apresente limitações, o estudo pode colaborar com o campo de conhecimento sobre a assistência prestada ao

\section{Referências}

ADESSE, L.; MONTEIRO, M. F. G; LEVIN, J.

Abortamento, um grave problema de saúde pública e de justiça social. Revista Radis - Comunicação em Saúde, Rio de Janeiro, n. 66. <Disponível em: <http://www6. ensp.fiocruz.br/radis/sites/default/files/radis_66.pdf $>$. Acesso em: 9 mar. 2008.

AQUINO, E. et al. Qualidade da atenção ao aborto no Sistema Único do Nordeste brasileiro: o que dizem as mulheres? Ciência \& Saúde Coletiva, Rio de Janeiro, v. 17, n. 7, p. 1765-1776, 2012.

BANKOLE, A.; SINGH, S.; HAAS, T. Characteristics of women who obtain induced abortion: a worldwide review. Internacional Family Planning Perspectives, New York, v. 25, n. 2, p. 68-77, 1999.

BRASIL. Ministério da Saúde. Secretaria de Atenção à Saúde. Departamento de Ações Programáticas Estratégicas. Atenção humanizada ao abortamento: norma técnica. 2. ed. Brasília, DF: Ministério da Saúde, Área Técnica da Mulher, 2011.

Ministério da Saúde. Secretaria de Atenção à Saúde. Departamento de Ações Programáticas Estratégicas. Área técnica da Mulher. Protocolo do misoprostol. 2012. Disponível em: <http://bvsms.saude. gov.br/bvs/publicacoes/protocolo_utilizacao_misoprostol_obstetricia.pdf>. Acesso em: 6 ago. 2015.

DINIZ, D.; MEDEIROS, M. Aborto no Brasil: uma pesquisa domiciliar com técnica de urna. Ciência \&t Saúde Coletiva, Rio de Janeiro, v. 15, supl. 1, p. 959-966, 2010.

FUSCO, C. L. B; ANDREONI, S.; SILVA, R. S.

Epidemiologia do aborto inseguro em uma população abortamento. A qualificação do preenchimento das fichas pelos profissionais de saúde - desde as informações sobre a queixa principal, a evolução clínica e cirúrgica, e o diagnóstico de saída - é primordial, tanto para um melhor conhecimento sobre as complicações do abortamento e a assistência prestada quanto para a utilização de dados reais e menos estimados sobre a realidade das mulheres que abortam. em situação de pobreza - Favela Inajar de Souza. Rev. bras. Epidemiol., São Paulo, v. 11, n. 1, p. 78-88, 2008.

HEILBORN, M. L. et al. Gravidez imprevista e aborto no Rio de Janeiro, Brasil: gênero e geração nos processos decisórios. Sex Saúde Soc., Rio de Janeiro, n. 12, 2011.

MARIUTI, M. G. et al. Abortamento: um estudo da morbidade hospitalar no país. Revista Brasileira de Medicina, São Paulo, v. 67, n. 4, p. 97-103, 2010.

MENEZES, G.; AQUINO, E. M. L. Pesquisa sobre o aborto no Brasil: avanços e desafios para o campo da saúde coletiva. Cad. Saúde Pública, Rio de Janeiro, v. 25 supl. 2, 2009.

ORGANIZAÇÃO MUNDIAL DA SAÚDE (OMS). Abortamento seguro: orientação técnica e de políticas para sistemas de saúde. 2 ed. Genebra: OMS, 2013.

OSAVA, R. H. et al. Perfil reprodutivo e padrões de consumo do misoprostol (Cytotec) como droga abortiva em usuárias de baixa renda. Revista enfermagem UERJ, Rio de Janeiro, v. 5, n. 1, p. 325-330, 1997.

RAMOS, K. S.; FERREIRA, A. L. C. G.; SOUZA, A. I. Mulheres hospitalizadas por abortamento em uma Maternidade Escola na Cidade do Recife. Brasil. Rev Esc Enferm USP, São Paulo, v. 44, n. 3, p. 605-610, 2010.

RIO DE JANEIRO (Cidade). Prefeitura Municipal do Rio de Janeiro. Secretaria Municipal de Saúde e Defesa Civil. Gestão do Programa Cegonha Carioca - Módulo Acolhimento. Disponível em: <http://www.rio.rj.gov. br/dlstatic/10112/176388/DLFE-208307.../AnexoB. pdf $>$. Acesso em: 18 ago. 2013. 
SACILOTO, M. P. et al. Aspiração manual intrauterina no tratamento do abortamento incompleto até 12 semanas gestacionais: uma alternativa à curetagem uterina. Rev. bras. ginecol. obstet., Rio de Janeiro, v. 33, n. 10, p. 292-296, 2011.

SINGH, S. et al. Aborto a nível Mundial: que há cambiado? In: Aborto a nível mundial: uma década de progresso desigual. Nova Iorque: Allan Guttmacher Institute, 2010, p. 7-11.

SINGH, S.; MONTEIRO, M. F.; LEVIN, J. Trends in hospitalization for abortion-related complications in Brazil, 1992-2009: Why the decline in numbers and severity? International journal of gynaecology and Obstetrics, Edmond, n. 118, p. 99-106, 2012.
VICTORA, C. G. et al. A saúde das mães e crianças no Brasil: progressos e desafios. The Lancet, Londres, $\mathrm{p}$. 32-46, maio 2011. (Saúde no Brasil, 2).

VIEIRA, L. M. et al. Abortamento na adolescência: um estudo epidemiológico. Ciência \&t Saúde Coletiva, Rio de Janeiro, v. 12, n. 5, p. 1201-1208, 2007.

WEBER, D. Brasil em $128^{\circ}$ em ranking de gravidez precoce. O Globo. Disponível em: <www.oglobo.com. br>. Acesso em: 10 mar. 2013.

Recebido para publicação em junho de 2014

Versão final em julho de 2015

Conflito de interesses: inexistente

Suporte financeiro: não houve 